\title{
Alcohol Alters Men's Perceptual and Decisional Processing of Women's Sexual Interest
}

\author{
Coreen Farris \\ Indiana University
}

\author{
Teresa A. Treat \\ Yale University
}

\author{
Richard J. Viken \\ Indiana University
}

\begin{abstract}
The current investigation examines the etiology of men's errors in sexual perception after moderate alcohol use. Sensitivity and bias estimates, derived from multidimensional signal detection analysis, revealed that men's alcohol-influenced performance was associated with declining sensitivity to the distinction between women's friendliness and sexual interest. However, sensitivity to the distinction between conservative and provocative clothing was unaffected. Similarly, an alcohol dose led to an increased bias to respond that women's ambiguous cues were sexual interest (rather than friendliness) but did not influence response thresholds for clothing style. Thus, there was specificity to the perceptual and decisional changes associated with alcohol use rather than a simple degradation of men's capacity to process all dating-relevant cues in the environment. Given the link between alcohol use, sexual misperception, and acquaintance-initiated sexual coercion, understanding the etiology of sexual misperception in the context of alcohol use may inform sexual coercion prevention efforts.
\end{abstract}

Keywords: alcohol, social perception, sexual misperception, signal detection

Alcohol is readily available in typical early dating contextsuniversity parties, restaurants, clubs, and bars-and it has been linked to first-time, consensual sex between new partners (Cooper \& Orcutt, 1997; Prince \& Bernard, 1998). Unfortunately, alcohol use is also associated with acquaintance-initiated sexual violence. For over half of reported attempted or completed rapes among university students, alcohol use by one or both individuals is reported (Abbey et al., 2002). Abbey and colleagues have theorized that the link between alcohol and sexual violence is partially mediated by errors in sexual perception (Abbey, McAusland, \& Ross, 1998). That is, a man's alcohol use may increase the probability that he will misperceive a woman's nonverbal behavior as indicative of sexual interest rather than simple friendliness. Misperception of friendliness as sexual interest, in turn, predicts increased risk for sexual violence (see Farris, Treat, Viken \& McFall, 2008b, for a review). In the current article, we investigate the first component of this model: By what perceptual or decisional processes does alcohol intoxication interfere with the accurate interpretation of dating-relevant cues?

Basic research establishes that alcohol use impairs cognitive functions such as selective attention (Abroms \& Fillmore, 2004), cognitive control (Fillmore, Marczinski, \& Bowman, 2005), and

Coreen Farris and Richard J. Viken, Psychological and Brain Sciences, Indiana University; Teresa A. Treat, Department of Psychology, Yale University.

Coreen Farris is now at the Division of General Internal Medicine, University of Pittsburgh School of Medicine.

Correspondence concerning this article should be addressed to Coreen Farris, University of Pittsburgh, Parkvale 310, 200 Meyran Avenue, Pittsburgh, PA 15213. E-mail: farrisca@upmc.edu information processing (Schweizer et al., 2006). Given that these basic processes lie upstream from performance on more complex social perception tasks, it should not come as a surprise that alcohol use is also associated with changes in performance on dating-relevant social tasks. In laboratory manipulations, acute alcohol intoxication increases the likelihood that men will judge a female confederate's behavior to be sexually suggestive (Abbey, Zawacki, \& Buck, 2005), which may be related to alcohol-related changes in perceptual processing. In an investigation that examined men's processing of photographs of women that varied along dimensions such as provocativeness, affect, appeal, and attractiveness, Treat and colleagues found that relative to sober performance, alcohol use was associated with significantly greater variance estimates in a probabilistic multidimensional scaling solution (Treat, McFall, Viken, Nosofsky, et al., 2002). That is, alcohol was related to larger individual differences in perception and/or greater difficulty or confusion in comparing and contrasting images in the stimulus set. The current investigation is designed to clarify further the processing changes associated with alcohol-related errors in sexual perception.

One possible source of error may be an increased reliance on nondiagnostic, or universal, cues to make decisions about a woman's sexual interest. Universal cues refer to information that is visible to all individuals in the social setting (e.g., choice of clothing, youthfulness, hair style). These cues are not person specific; that is, any one perceiver cannot conclude, using only information such as a woman's clothing choice, that the woman is sexually interested in the perceiver specifically. Person-specific cues, in contrast, are directed to one individual in particular to communicate interest or lack of interest in a dating or sexual relationship with that specific person. These person-specific cues 
include facial affect, verbal cues, posture, and physical proximity. In general, it would be expected that person-specific cues explain more variance in correct judgments of sexual interest than do universal cues. To the extent that alcohol interferes with an individual's ability to direct attention to person-specific cues (e.g., facial affect) rather than universal cues (e.g., provocative clothing), he will be increasingly likely to misperceive sexual intent.

Although it often is suggested that observed alcohol-related errors can be traced to an increased bias to focus on sexual interest cues (Abbey et al., 2005), the typical measurement schemes cannot support such a claim. There are two primary cognitive mechanisms by which alcohol could interfere with accurate judgment of sexual intent, but the source of the error has never been decomposed. One possibility, which has been theorized but never tested, is that a decisional source of error, or bias, shifts intoxicated men's threshold for selecting one response over another (e.g., sexual interest over friendliness) relative to sober men. An alternative explanation is that a perceptual source of error, or insensitivity, produces a decline in the perceiver's ability to discriminate between categories (e.g., friendliness vs. sexual interest). This distinction has been central to investigations of simple perception in cognitive science, which provides the computational models necessary to decompose sources of error for this application.

The present study uses multidimensional signal detection analyses (MSDA; Kadlec \& Townsend, 1992) in two ways: (a) to parse perceptual (sensitivity) from decisional (bias) explanations for alcohol's effects on men's perception of women's sexual interest cues and (b) to examine alcohol-related effects on processing of person-specific affective cues versus universal clothing style cues. ${ }^{1}$ Given that alcohol-intoxicated men, as compared with sober men, rate women as more sexually interested and disinhibited (Abbey et al., 2005; Abbey, Zawacki, \& McAuslan, 2000), we predicted that processing of diagnostic person-specific cues would be affected by alcohol use. Universal clothing style cues represent impelling but largely nondiagnostic cues and hence provide a useful, dating-relevant counterpoint to affective processing. Inclusion of a clothing style discrimination also allows an examination of the specificity versus generality of observed alcohol effects. There is no empirical work to guide predictions about whether observed changes in processing of either affect or clothing style will be attributed to decisional bias, perceptual sensitivity, or a combination of the two processes. Thus, we approached the decomposition of these processes from an exploratory perspective.

\section{Method}

\section{Participants}

Eligible participants, recruited via flyer, were male, heterosexual, and 21 years old or older. Participants were required to report that they drank alcohol at least one time per month and had experience drinking four or more standard drinks in one drinking episode at least one time in the past year. Eligibility was evaluated during a screening interview via telephone that included demographics, medical questions (to ensure that alcohol use was not medically contraindicated), and the Michigan Alcohol Screening Test (Seltzer, 1971). Participants with a Michigan Alcohol Screening Test score that indicated possible problems with alcohol use $(>5)$ were not eligible to participate.
A total of 59 men participated, of whom $89.8 \%$ were Caucasian. Most participants were young $(M=22.7$ years old, $S D=2.32)$ and many were university students $(69.5 \%)$. They reported drinking an average of 6.6 standard alcoholic drinks $(S D=3.43)$ on 10.4 occasions per month $(S D=5.12)$.

\section{Identification Task}

Stimulus set. Extensive pilot testing was conducted to develop a set of images that varied on the dimensions of affect (friendly to sexually interested) and clothing style (conservative to provocative). The original stimulus set contained 1,127 full-body photographs of undergraduate women, which were rated by 497 undergraduate men on a number of scales (including friendly, sexually interested, provocatively dressed, and attractive, among others). Images rated above the mean for the relevant category and below the mean on all other categories were retained for possible inclusion. Next, these images were included in a forced-choice task completed by a unique sample of 80 undergraduate men and women. After this round, photographs were considered if the majority of men and the majority of women identified the target as belonging to the same category. Final image selection was balanced to ensure that half of the targets in each category were rated below the midpoint of the provocatively dressed scale (i.e., conservatively dressed) and half were rated above the midpoint (i.e., provocatively dressed). Normative attractiveness ratings were equivalent across the four stimulus types. The final image set contained 70 sexually interested targets and 70 friendly targets. Half of the targets in each category were provocatively dressed.

Although undergraduates' ratings of the stimuli determined classification of women's affect and clothing style, examination of the selected stimuli suggests that specific stimulus features frequently distinguished classification membership for both dimensions. Targets classified as conservatively dressed typically wore less revealing outfits, such as a pair of jeans and a sweatshirt. Provocatively dressed targets wore more revealing clothing that they might wear to a party or dancing, such as a short skirt and form-fitting top. Targets judged to be sexually interested tended to tilt their head, placed their weight more on one foot than another (emphasizing one hip), and made sidelong eye contact or smiled slightly. Targets judged to be friendly tended to face directly forward with their weight placed evenly on both feet while smiling broadly without tilting their head.

Task. Participants viewed each target on a computer screen for 1,000 ms and responded to two questions serially: "Was the woman provocatively or conservatively dressed?" and "Was the woman displaying sexual interest or friendliness?" Question order was counterbalanced across participants. One hundred forty unique targets were repeated across three blocks for a total of 420 trials. Presentation order was randomized within block.

Relative difficulty. D prime $\left(d^{\prime}\right)$ is a signal detection parameter (Green \& Swets, 1966) that measures the difficulty an observer has discriminating between two categories. In a sample of 504 undergraduate men, participants' average sensitivity to the

\footnotetext{
${ }^{1}$ See Macmillan and Creelman (2004) for greater detail on detection theory in general, Kadlec and Townsend (1992) for an introduction to MSDA, and Farris, Viken, and Treat (in press) for a tutorial on and clinical application of MSDA.
} 
distinction between friendliness and sexual interest in the same stimulus set was $1.39(S D=0.56)$, and participants' average sensitivity to the distinction between clothing styles was 1.32 $(S D=0.37$; Farris, Viken, \& Treat, 2009). The values indicate that both tasks are moderately difficult and that for this stimulus set, undergraduate men find it slightly more difficult to discriminate between clothing styles than between affect categories, $t(503)=$ $2.78, p<.01$, Cohen's $d=0.15$.

\section{Design and Procedure}

Participants attended separate laboratory sessions to complete the identification task once with and once without an alcohol dose. Sessions were scheduled one week apart, each session began between 1:30 and 2:30 p.m., and the session order (with or without alcohol) was counterbalanced across subjects. Participants were instructed to eat a light lunch at 12:00 p.m. and to refrain from drinking alcohol for $24 \mathrm{hr}$ prior to each session.

Identification task with an alcohol dose. During the alcohol session, participants received a dose of $0.7 \mathrm{~g} / \mathrm{kg}$ ethanol calculated to produce an average peak breath alcohol concentration (BrAC) of $.06 \%$. Drinks were 100-proof vodka mixed at a 1:3 ratio with fruit juice. Participants consumed the dose over $20 \mathrm{~min}$; this was followed by a 10-min absorption period. The identification task began after the absorption period.

Identification task without an alcohol dose. With the exception of excluding alcohol from the beverage, procedures identical to those used during the alcohol session were followed during the juice-only session. The quantity of juice was unchanged across sessions. Participants were not deceived about the alcohol content of their beverages.

\section{MSDA}

Marginal sensitivity. Across-category estimates of sensitivity measure the perceptual distance between two levels of one dimension, holding the level of the second dimension constant. For example, $\mathrm{d}^{\prime}{ }_{\mathrm{AFF} \mid \mathrm{CD}}$ estimates the perceptual distance between the means of the two affect categories (friendly and sexually interested) for conservatively dressed targets. It would be computed as follows:

$$
d^{\prime}{ }_{\mathrm{AFF} \mid \mathrm{CD}}=z\left[P\left(R_{\mathrm{SI}} \mid \mathrm{SI} . \mathrm{CD}\right)\right]-z\left[P\left(R_{\mathrm{SI}} \mid \mathrm{FR} . \mathrm{CD}\right)\right],
$$

where $z$ is the inverse of the normal distribution function. A sensitivity estimate of zero indicates that the perceptual distributions overlap perfectly and the participant is wholly unable to distinguish between the two target categories, and it increases positively as discrimination between the features improves.

Marginal bias. The marginal bias parameters provide estimates of four decisional thresholds corresponding to the points along the two dimensions at which the participant divides the perceptual space. For example, among conservatively dressed targets, as the perceptual dimension for affect increases from clearly friendly toward sexual interest, $\beta_{\mathrm{AFF} \mid \mathrm{CD}}$ represents the point at which the participant switches from a friendly to a sexually interested response. Mathematically,

$$
\beta_{\mathrm{AFF} \mid \mathrm{CD}}=\frac{\phi\left\{z\left[P\left(R_{\mathrm{SI}} \mid \mathrm{SI} . \mathrm{CD}\right)\right]\right\}}{\phi\left\{z\left[P\left(R_{\mathrm{SI}} \mid \mathrm{FR} \cdot \mathrm{CD}\right)\right]\right\}},
$$

where $\Phi$ is the height of the normal density function. All marginal $\beta$ estimates are computed similarly. The bias parameter $B$ was natural $\log$ transformed to approximate a normal distribution for later statistical analyses. Given equal presentation probabilities, $\ln (B)$ is equal to 0 when the participant is responding optimally (i.e., is not biased toward either response), becomes increasingly negative as the decisional boundary becomes more lenient (e.g., more biased to respond that the target intended to signal sexual interest), and becomes increasingly positive as the decisional boundary becomes more conservative (e.g., more biased to respond that the target intended to signal friendliness). The bias estimate is free to vary independently from the sensitivity estimate.

\section{Analytic Strategy}

MSDA estimates of sensitivity and bias were used to examine differences in participants' perceptual and decisional processing with and without an alcohol dose. A general linear model approach was used for statistical analyses of these parameter estimates. The models included the within-session, within-person experimental factors of intent (friendly vs. sexually interested) and clothing style (conservative vs. provocative); the across-session, within-person factor of alcohol dose (alcohol vs. juice only); an across-person factor for alcohol session order (alcohol session conducted during the first or second session); and all interactions. Marginal sensitivity and marginal bias were treated as repeated within-person, within-session measures.

\section{Results}

\section{Manipulation Checks}

During the alcohol session, after the absorption period, the average $\mathrm{BrAC}$ was $.054 \%(S D=.014)$. Average BrAC readings following the first, second, and third block of trials were $.056 \%$ $(S D=.014), .051 \%(S D=.012)$, and $.050 \%(S D=.012)$, respectively. As in previous research, during sober performance, the discrimination between clothing styles $\left(d^{\prime}=1.49, S D=0.44\right)$ was moderately more difficult than the discrimination between affect categories $\left(d^{\prime}=1.64, S D=0.55\right), t(58)=2.49, p<.05$, Cohen's $d=0.31$.

\section{Perceptual and Decisional Contributions to Detection of Affect}

The analysis revealed a main effect of alcohol, $F(1,57)=6.61$, $p<.05, \eta_{\mathrm{p}}^{2}=.104$. Alcohol was associated with a change in participants' sensitivity and bias on the affect dimension. That is, compared with their own performance without an alcohol dose $\left(d^{\prime}=1.64, S D=0.56\right)$, participants performing the identification task after consuming alcohol were significantly less sensitive to differences in target women's affect $\left(d^{\prime}=1.50, S D=0.57\right), F(1$, $57)=6.13, p<.05, \eta_{\mathrm{p}}^{2}=.097$. Participants also adopted a more lenient threshold for labeling women's positive affect sexual interest after alcohol consumption, $\ln (\beta)=0.65, S D=0.94$, compared with the threshold they used without an alcohol dose, $\ln (\beta)=0.93, S D=1.34, F(1,57)=5.29, p<.05, \eta_{\mathrm{p}}^{2}=.085$.

Finally, there was an interaction indicating that the effect of clothing style differed for the repeated measures of bias and 
sensitivity to affect, $F(1,57)=4.70, p<.05, \eta_{\mathrm{p}}^{2}=.076$. Analyses revealed that clothing style had no influence on bias estimates for affect, $F(1,58)=.004, p=n s, \eta_{\mathrm{p}}^{2}=.000$. However, clothing style significantly influenced participants' sensitivity to affect, $F(1$, $58)=16.00, p<.001, \eta_{\mathrm{p}}^{2}=.216$. Participants were better able to distinguish friendliness from sexual interest when target women were dressed provocatively, $\left(d^{\prime}=1.66, S D=0.61\right)$ rather than conservatively $\left(d^{\prime}=1.48, S D=0.48\right)$. No other main or higher order effects significantly predicted participants' judgments about women's affect cues.

\section{Perceptual and Decisional Contributions to Detection of Clothing Style}

Participants' identification of clothing style differences was not significantly influenced by alcohol, $F(1,57)=0.621, p=n s$, $\eta_{\mathrm{p}}^{2}=.011$. That is, alcohol influenced neither sensitivity nor bias estimates for clothing style. There was a main effect of affect, $F(1$, $57)=8.61, p<.05, \eta_{\mathrm{p}}^{2}=.081$. Analysis of sensitivity estimates revealed that participants were more sensitive to clothing style when affect was friendliness $\left(d^{\prime}=1.60, S D=0.48\right)$ rather than sexual interest $\left(d^{\prime}=1.31, S D=0.41\right), F(1,57)=18.28, p<.001$, $\eta_{\mathrm{p}}^{2}=.243$. Descriptive statistics of the clothing style bias parameters suggested that participants were more biased to respond that clothing was provocative when affect was sexual interest, $\ln (\beta)=$ $1.14, S D=1.10$, rather than friendliness, $\ln (\beta)=1.38, S D=1.47$, but this difference was not statistically significant, $F(1,57)=$ $1.89, p=n s, \eta_{\mathrm{p}}^{2}=.032$. No other main or higher order effects predicted participants' perceptual and decisional processing of women's clothing styles.

\section{Discussion}

Relative to their own performance sober, men's alcoholinfluenced performance was marked by declining sensitivity to women's affective cues, such that they were less able to discriminate friendliness from sexual interest. This decreased sensitivity may serve to increase the probability of risky social errors, particularly in situations involving alcohol use. However, sensitivity estimates for clothing style remained unchanged whether men were performing with or without alcohol. Given that the average $d^{\prime}$ values suggest that clothing cues were more difficult to discriminate than affect cues, alcohol-related change in sensitivity to affect, but not to clothing style, cannot be discarded as merely a function of differences in task difficulty. It appears that the influence of moderate alcohol consumption was specific (interfering with judgments of dating-relevant affect) rather than general (degrading an individual's capacity to discriminate effectively between all cues in the environment). It is particularly problematic that alcohol degrades processing of a person-specific cue given its close tie to women's responsiveness to sexual advances.

The results contribute to an emerging framework of process model investigations of sexual perception (Treat, McFall, Viken, \& Krushke, 2001). Moderate alcohol consumption is situated within a group of situational and attitudinal variables that affect sexual perception, serving to decrease sensitivity to the subtle cues that differentiate women's sexual interest from platonic interest. Endorsement of attitudes that support rape and blame women for victimization as well as self-reported history of sexual coercion are associated with lower sensitivity to women's dating-relevant affect (Farris, Viken, Treat, \& McFall, 2006; Farris et al., 2009). However, these effect sizes are an order of magnitude lower than the influence of moderate alcohol use on men's perception, suggesting that alcohol may be a particularly important variable to consider. Gender is a particularly strong effect; relative to women, men find it more difficult to discriminate between women's affective cues (Farris, Treat, Viken, \& McFall, 2008a). To the extent that these processes are additive, alcohol use may be particularly problematic among high-risk men who already hold attitudes associated with risk for perpetration of sexual violence.

Alcohol consumption was also associated with a change in marginal bias estimates for affect but not clothing style. Relative to their own sober performance, participants were more likely to respond that affect was sexual interest rather than friendliness. This decline in the threshold for detecting sexual interest will ensure that a greater proportion of sexually interested women will be correctly identified and thus may increase the probability that an intoxicated individual will pursue an interested partner. However, any improvement in detecting interested women that is attributed to a shift in bias will also increase the likelihood of misidentifying a friendly woman as sexually interested. It is precisely this type of social error that is associated with increased risk for sexual coercion. An alcohol-related shift in the decisional threshold may be one reason why ambiguous positive cues are more likely to be misidentified as sexual interest during drinking situations (Abbey et al., 2005). Although affect bias estimates shifted significantly relative to sober performance, the threshold for detecting provocative clothing was not influenced by alcohol consumption. Some decisional processes remained intact after a moderate alcohol dose.

It is interesting that the influence of alcohol in shifting men's decisional thresholds downward appears to be unique. Gender and sexual aggression status do not predict this shift (Farris et al., 2006, 2008a). That is, men are no more likely than women to use lenient thresholds for labeling positive affect sexual interest (rather than friendliness), and, compared with nonaggressive men, men with histories of sexual aggression are no more or less lenient in detecting sexual interest. However, other situational factors do seem to play a role in predicting bias, particularly the provocativeness of women's clothing. Men's thresholds for detecting sexual interest drop significantly when women are dressed provocatively; perceivers become more likely to respond that women's positive affect cues signal sexual interest (rather than friendliness; Farris et al., 2006, 2008a).

From an applied perspective, an understanding of the processes responsible for alcohol-related sexual misperception could lead to intervention strategies to reduce the risk of misperception and, we hope, in turn, sexual coercion. Parsing perceptual from decisional sources of error is an important first step in directing the form of retraining. A rich basic cognitive science literature demonstrates that shifting sensitivity and bias requires different strategies. Sensitivity can be improved via repeated learning trials with feedback. For example, the individual might identify women's personspecific cues and then receive feedback about whether his decision was correct. Bias is often shifted by changing the costs for certain types of incorrect decisions. Here, the cost for mistakenly identifying sexual interest may need to increase, perhaps by highlighting the potential social and even legal consequences of pursuing an 
uninterested partner. The clear influence of alcohol use on both forms of processing suggests that it will be important to target both processes and to ensure that retrained processing is robust in the face of moderate alcohol intoxication. It may be necessary to train perceptual processing during both sobriety and periods of intoxication to ensure generalizability to dating situations that include alcohol use.

Just as the cognitive processes that lie upstream from observed errors in sexual perception can be complex, so too is the understanding of alcohol effects in general. The alcohol dosing procedure used in the current investigation allows general inferences about the influence of alcohol on sexual perception but does not allow us to determine the extent to which these effects are due to alcohol pharmacology or expectancies (Brown, Goldman, Inn, $\&$ Anderson, 1980). Sexual decision making is clearly one arena in which expectancies about the influence of alcohol play an important role (Wilson, Calhoun, \& McNair, 2002). An important future investigation will parse the relative contribution of expectancy versus pharmacological effects of alcohol on men's perception of women. In addition, alcohol effects are clearly dose dependent and are also sensitive to whether a given BrAC is measured on the ascending or descending limb of alcohol intoxication (Kalant, 1996). Currently, the functional form of the relationship between alcohol dose, alcohol limb, and processing of women's affect cues is still unclear. Finally, non- and light drinkers may respond to a moderate alcohol dose differently than the relatively heavydrinking sample examined here would respond to the same dose.

The identification paradigm required participants to judge women's sexual intent from relatively impoverished stimuli with limited processing time. Social and sexual communication more often occur in a dynamic and reciprocal interchange between actors. Perceivers may accumulate information over time about a potential partner's interest in order to change or refine interpretation of her behavior. Thus, it will be important to explore perceptual and decisional processing of sexual intent cues in response to increasingly rich stimuli, such as videotaped vignettes or scripted, live interactions, to establish the stability of the findings. At the same time, first-impression judgments, such as those captured by short exposure to photographs, also influence social perception across longer time scales and thus merit continued consideration (Ambady, Bernieri, \& Richeson, 2000).

The current investigation extended prior work by demonstrating a unique effect of alcohol on processing of sexual interest cues, with the observed changes attributable to both declining sensitivity to affective cues and a shift in bias toward more liberal thresholds for detecting sexual interest. It is interesting that alcohol did not interfere with the processing of all aspects of women: Discriminating provocative clothing from conservative clothing was preserved and bias for detecting provocative clothing was unchanged. Understanding the precise mechanisms by which alcohol serves to change social perception may provide a unique opportunity to intervene to remediate deficits. Future work testing plausible interventions and their effect on real-world social perception would be a useful extension to the current research. Finally, the research contributes to a growing literature that documents the utility of merging the methodological advances in cognitive science with previously unexamined theoretical questions in clinical and social psychology (Neufeld, 2007).

\section{References}

Abbey, A., McAuslan, P., \& Ross, L. T. (1998). Sexual assault perpetration by college men: The role of alcohol, misperception of sexual intent, and sexual beliefs and experiences. Journal of Social and Clinical Psychology, 17, 167-195.

Abbey, A., Zawacki, T., \& Buck, P. O. (2005). The effects of past sexual assault perpetration and alcohol consumption on men's reactions to women's mixed signals. Journal of Social and Clinical Psychology, 24, $129-155$.

Abbey, A., Zawacki, T., Buck, P. O., Testa, M., Parks, K., Norris, J., . . . Martell, J. (2002). How does alcohol contribute to sexual assault? Alcoholism: Clinical and Experimental Research, 26, 575-584.

Abbey, A., Zawacki, T., \& McAuslan, P. (2000). Alcohol's effect on sexual perception. Journal of Studies on Alcohol, 61, 688-697.

Abroms, B. D., \& Fillmore, M. T. (2004). Alcohol-induced impairment of inhibitory mechanisms involved in visual search. Experimental and Clinical Psychopharmacology, 12, 243-250.

Ambady, N., Bernieri, F. J., \& Richeson, J. A. (2000). Toward a histology of social behavior: Judgmental accuracy from thin slices of the behavioral stream. Advances in Experimental Social Psychology, 32, 201-271.

Kalant, H. (1996). Pharmacokinetics of ethanol: Absorption, distribution, and elimination. In H. Begleiter \& B. Kissin (Eds.), The pharmacology of alcohol and alcohol dependence (pp. 15-58). New York: Oxford University Press.

Brown, S. A., Goldman, M. S., Inn, A., \& Anderson, L. (1980). Expectations of reinforcement from alcohol: Their domain and relation to drinking patterns. Journal of Consulting and Clinical Psychology, 48, 419-426.

Cooper, M. L., \& Orcutt, H. K. (1997). Drinking and sexual experience on first dates among adolescents. Journal of Abnormal Psychology, 106, 191-202.

Farris, C., Treat, T. A., Viken, R. J., \& McFall, R. M. (2008a). Perceptual mechanisms that characterize gender differences in decoding women's sexual intent. Psychological Science, 19, 348-354.

Farris, C., Treat, T. A., Viken, R. J., \& McFall, R. M. (2008b). Sexual coercion and the misperception of sexual intent. Clinical Psychology Review, 28, 48-66.

Farris, C., Viken, R. J., \& Treat, T. A. (2009). [Sensitivity to affect and clothing style]. Unpublished raw data.

Farris, C., Viken, R. J., \& Treat, T. A. (in press). Perceived association between diagnostic and non-diagnostic cues of women's sexual interest: General recognition theory predictors of risk for sexual coercion. Journal of Mathematical Psychology.

Farris, C., Viken, R. J., Treat, T. A., \& McFall, R. M. (2006). Choice theory measures of social perception: An application to sexual coercion. Psychological Science, 17, 869-875.

Fillmore, M. T., Marczinski, C. A., \& Bowman, A. M. (2005). Acute tolerance to alcohol effects on inhibitory and activational mechanisms of behavioral control. Journal of Studies on Alcohol, 66, 663-672.

Green, D. M., \& Swets, J. A. (1966). Signal detection theory and psychophysics. New York, NY: Wiley.

Kadlec, H., \& Townsend, J. T. (1992). Signal detection analyses of dimensional interactions. In F. G. Ashby (Ed.), Multidimensional models of perception and cognition (pp. 181-231). Hillsdale, NJ: Erlbaum.

Macmillan, N. A., \& Creelman, C. D. (2004). Detection theory: A user's guide. Hilldale, NJ: Erlbaum

Neufeld, R. W. J. (2007). Advances in clinical cognitive science: Formal modeling of processes and symptoms. Washington, DC: American Psychological Association.

Prince, A., \& Bernard, A. L. (1998). Alcohol use and safer sex behaviors of students at a commuter university. Journal of Alcohol and Drug Education, 43, 1-19.

Schweizer, T. A., Vogel-Sprott, M., Danckert, J., Roy, E. A., Skakum, A., \& Broderick, C. E. (2006). Neuropsychological profile of acute alcohol 
intoxication during ascending and descending blood alcohol concentrations. Neuropsychopharmacology, 31, 1301-1309.

Seltzer, M. (1971). The Michigan Alcoholism Screening Test: A quest for a new diagnostic instrument. American Journal of Psychiatry, 127, 1653-1658.

Treat, T. A., McFall, R. M., Viken, R., J., Nosofsky, R. M., MacKay, D. B., \& Krushke, J. K. (2002). Assessing clinically relevant perceptual organization with multidimensional scaling techniques. Psychological Assessment, 14, 239-252.

Treat, T. A., McFall, R. M., Viken, R. J., \& Krushke, J. K. (2001). Using cognitive science methods to assess the role of social information processing in sexually coercive behavior. Psychological Assessment, 13, $549-565$.

Wilson, A. E., Calhoun, K. S., \& McNair, L. D. (2002). Alcohol consumption and expectancies among sexually coercive men. Journal of Interpersonal Violence, 17, 1145-1159.

Received February 25, 2009

Revision received October 22, 2009

Accepted October 30, 2009 